\title{
Some Domination Parameters of Direct Product Graphs of Cayley Graphs with Arithmetic Graphs
}

\author{
S. Uma Maheswari \\ Lecturer \\ Department of Mathematics \\ JMJ College For Women \\ Tenali, AP, India
}

\author{
B. Maheswari \\ Professor \\ Department of \\ Applied Mathematics \\ S.P. Women's University \\ Tirupati, AP, India
}

\author{
M. Manjuri \\ Department of \\ Applied Mathematics \\ S.P. Women's University \\ Tirupati, AP, India
}

\begin{abstract}
Number Theory is one of the oldest branches of mathematics, which inherited rich contributions from almost all greatest mathematicians, ancient and modern.

Nathanson [1] paved the way for the emergence of a new class of graphs, namely Arithmetic Graphs by introducing the concepts of Number Theory, particularly, the Theory of Congruences in Graph Theory. Cayley graphs are another class of graphs associated with the elements of a group. If this group is associated with some arithmetic function then the Cayley graph becomes an Arithmetic graph. Inspired by the interplay between Number Theory and Graph Theory several researchers in recent times are carrying out extensive studies on various Arithmetic graphs in which adjacency between vertices is defined through various arithmetic functions.
\end{abstract}

In this paper, we consider direct product graphs of Cayley graphs with Arithmetic graphs and present some domination parameters of these graphs.

\section{Keywords}

Dominating set, Total Dominating set, Euler Totient Cayley Graph, Arithmetic $V_{n}$ graph, Direct Product Graph.

AMS (MOS) Subject Classification: 6905c

\section{INTRODUCTION}

Domination in graphs has been an extensively research branch of graph theory. (For more details refer [2, 3]). Dominating sets play an important role in practical applications, such as allocation of re-hydrants or serving sites of other supplies, modeling of relations in human groups or animal biotopes. They have applications in diverse areas such as logistics and networks design, mobile computing, resource allocation and telecommunication. Cayley graphs are excellent models for interconnection networks, investigated in connection with parallel processing and distributed computation.

In this section we present necessary definitions, observations and some useful results that we need for next sections.

\section{DOMINATING SET}

Let $G$ be a graph with vertex set $V$. A subset $D$ of $V$ is said to be a dominating set of $G$ if every vertex in $V-D$ is adjacent to a vertex in $D$.

The minimum cardinality of a dominating set is called the domination number of $G$ and is denoted by $\gamma(G)$.

\section{TOTAL DOMINATING SET}

Let $G$ be a graph without isolated vertices. Then a total dominating set $T$ is a subset of vertex set $V$ such that every vertex of $V$ is adjacent to some vertex in $T$.

The minimum cardinality of a total dominating set of $G$ is called the total domination number of $G$ and is denoted by $\gamma_{t}(G)$.

\section{DIRECT PRODUCT GRAPHS}

In the literature, the direct product is also called as the tensor product, categorical product, cardinal product, relational product, Kronecker product, weak direct product, or conjunction. As an operation on binary relations, the tensor product was introduced by Alfred North Whitehead and Bertrand Russell in their Principia Mathematica [4]. It is also equivalent to the Kronecker product of the adjacency matrices of the graphs given by Weichsel [5].

If a graph can be represented as a direct product, then there may be multiple different representations (direct products do not satisfy unique factorization) but each representation has the same number of irreducible factors. Wilfried Imrich [6] gives a polynomial time algorithm for recognizing tensor product graphs and finding a factorization of any such graph.

This product is commutative and associative in a natural way (refer [7] for a detailed description on product graphs).

Let $G_{1}$ and $G_{2}$ be two simple graphs with their vertex sets as $V_{1}=\left\{u_{1}, u_{2}, \ldots, u_{l}\right\}$ and $V_{2}=\left\{v_{1}, v_{2}, \ldots, v_{m}\right\}$ respectively. Then the direct product of these two graphs denoted by $G_{1} \times G_{2}$ is defined to be a graph with vertex set $V_{1} \times V_{2}$, where $V_{1} \times V_{2}$ is the Cartesian product of the sets $V_{1}$ and $V_{2}$ such that any two distinct vertices $\left(u_{1}, v_{1}\right)$ and $\left(u_{2}, v_{2}\right)$ of $G_{1} \times G_{2}$ are adjacent if $u_{1} u_{2}$ is an edge of $G_{1}$ and $v_{1} v_{2}$ is an edge of $G_{2}$.

The cross symbol $\times$, shows visually the two edges resulting from the direct product of two edges.

Now we consider the direct product graph of Euler totient Cayley graphs with Arithmetic $V_{n}$ graphs. The properties of these graphs are presented in [8]. We briefly present Euler totient Cayley graph and Arithmetic $V_{n}$ graph.

\section{EULER TOTIENT CAYLEY GRAPH $G\left(Z_{n}, \varphi\right)$ AND ITS PROPERTIES}

Madhavi [9] introduced the concept of Euler totient Cayley graphs and studied some of its properties.

For any positive integer $n$, let $Z_{n}=\{0,1,2, \ldots . n-1\}$. Then $\left(Z_{n}, \oplus\right)$, where, $\oplus$ is addition modulo $n$, is an abelian 
group of order $n$. The number of positive integers less than $n$ and relatively prime to $n$ is denoted by $\varphi(n)$ and is called Euler totient function. Let $S$ denote the set of all positive integers less than $n$ and relatively prime to $n$.

That is $S=\{r / 1 \leq r<n$ and $\operatorname{GCD}(r, n)=1\}$. Then $|S|=\varphi(n)$.

Now we define Euler totient Cayley graph as follows.

For each positive integer $n$, let $Z_{n}$ be the additive group of integers modulo $n$ and let $S$ be the set of all integers less than $n$ and relatively prime to $n$. The Euler totient Cayley graph $G\left(Z_{n}, \varphi\right)$ is defined as the graph whose vertex set $V$ is given by $Z_{n}=\{0,1,2, \ldots . n-1\}$ and the edge set is $E=\{(x, y) / x-y \in S$ or $y-x \in S\}$.

The domination parameters of these graphs are studied by Uma Maheswari [8] and we present some of the results which we need without proofs and can be found in [10].

Theorem 1.1: If $n$ is a prime, then the domination number of $G\left(Z_{n}, \varphi\right)$ is 1 .

Theorem 1.2: If $n$ is power of a prime, then the domination number of $G\left(Z_{n}, \varphi\right)$ is 2

Theorem 1.3: The domination number of $G\left(Z_{n}, \varphi\right)$ is 2 , if $n=2 p$ where $p$ is an odd prime.

Theorem 1.4: Suppose $n$ is neither a prime nor $2 p$. Let $n=p_{1}^{\alpha_{1}} p_{2}^{\alpha_{2}} \ldots \ldots . p_{k}^{\alpha_{k}}$, where $p_{1}, p_{2}, \ldots p_{k}$ are primes and $\alpha_{1}, \alpha_{2}, \ldots . \alpha_{k}$ are integers $\geq 1$. Then the domination number of $G\left(Z_{n}, \varphi\right)$ is given by $\gamma\left(G\left(Z_{n}, \varphi\right)\right)=\lambda+1$, where $\lambda$ is the length of the longest stretch of consecutive integers in $V$, each of which shares a prime factor with $n$.

Theorem 1.5: If $n$ is a prime, then the total domination number of $G\left(Z_{n}, \varphi\right)$ is 2 .

Theorem 1.6: If $n$ is power of a prime, then the total domination number of $G\left(Z_{n}, \varphi\right)$ is 2 .

Theorem 1.7: The total domination number of $G\left(Z_{n}, \varphi\right)$ is 4 , if $n=2 p$, where $p$ is an odd prime.

Theorem 1.8: Suppose $n$ is neither a prime nor $2 p$. Let $n=p_{1}^{\alpha_{1}} p_{2}^{\alpha_{2}} \ldots \ldots p_{k}^{\alpha_{k}}$ where $p_{1}, p_{2}, \ldots p_{k}$ are primes and $\alpha_{1}, \alpha_{2}, \ldots \alpha_{k}$ are integers $\geq 1$. Then the total domination number of $G\left(Z_{n}, \varphi\right)$ is given by $\gamma_{t}\left(G\left(Z_{n}, \varphi\right)\right)=\lambda+1$, where $\lambda$ is the length of the longest stretch of consecutive integers in $V$ each of which shares a prime factor with $n$.

\section{ARITHMETIC $V_{\boldsymbol{n}}$ GRAPH}

Vasumathi [11] introduced the concept of Arithmetic $V_{n}$ graphs and studied some of its properties.

Let $n$ be a positive integer such that $n=p_{1}^{\alpha_{1}} p_{2}^{\alpha_{2}} \ldots \ldots . . p_{k}^{\alpha_{k}}$. Then the Arithmetic $V_{n}$ graph is defined as the graph whose vertex set consists of the divisors of $n$ and two vertices $u, v$ are adjacent in $V_{n}$ graph if and only if $\operatorname{GCD}(u, v)=p_{i}$, for some prime divisor $p_{i}$ of $n$.

In this graph vertex 1 becomes an isolated vertex. Hence we consider Arithmetic graph $V_{n}$ without vertex 1 as the contribution of this isolated vertex is nothing when the properties of these graphs and enumeration of some domination parameters are studied.

Clearly, $V_{n}$ graph is a connected graph. Because if $n$ is a prime, then $V_{n}$ graph consists of a single vertex. Hence it is a connected graph. In other cases, by the definition of adjacency in $V_{n}$, there exist edges between prime number vertices and their prime power vertices and also to their prime product vertices. Therefore each vertex of $V_{n}$ is connected to some vertex in $V_{n}$.

The domination parameters of these graphs are studied by S.Uma Maheswari [8] and we present some of the results which we need without proofs and can be found in [12].

Theorem 1.9: If $n=p_{1}^{\alpha_{1}} p_{2}^{\alpha_{2}} \ldots \ldots . p_{k}^{\alpha_{k}}$, where $p_{1}, p_{2}$, $\ldots p_{k}$ are primes and $\alpha_{1}, \alpha_{2}, \ldots . \alpha_{k}$ are integers $\geq 1$, then the domination number of $G\left(V_{n}\right)$ is given by

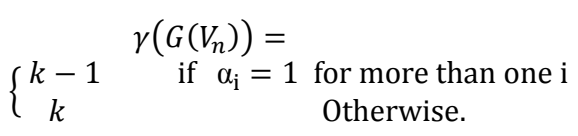

where $k$ is the core of $n$.

Theorem 1.10: Let $n=p_{1}^{\alpha_{1}} p_{2}^{\alpha_{2}} \ldots \ldots . p_{k}^{\alpha_{k}}$ where $\alpha_{\mathrm{i}} \geq 1$, $\forall i$. Then the total domination number of $G\left(V_{n}\right)$ is $k$, where $k$ is the core of $n$

\section{DOMINATION IN DIRECT PRODUCT GRAPH $G_{1} \times G_{2}$}

In this section we discuss the dominating sets of the direct product graph of Euler totient Cayley graph $G_{1}$ and Arithmetic $V_{n}$ graph $G_{2}$.

Theorem 2.1: If $n$ is a prime, then the domination number of $G_{1} \times G_{2}$ is $n$.

Proof: Suppose $n$ is a prime. Then $G_{1} \times G_{2}$ is a completely disconnected graph on $n$ vertices [13]. Since there is no edge between these $n$ vertices, all these vertices form a dominating set. Hence its domination number is $n$.

Theorem 2.2: If $n$ is power of a prime, then the domination number of $G_{1} \times G_{2}$ is 4 .

Proof: Let $n=p^{\alpha}$. Consider the graph $G_{1} \times G_{2}$.

Let $\begin{aligned} V\left(G_{1}\right) & =\left\{0,1,2,3, \ldots \ldots, p^{\alpha}-1\right\}=V_{1} \\ V\left(G_{2}\right) & =\left\{p, p^{2}, \ldots \ldots p^{\alpha}\right\}=V_{2} \text { and }\end{aligned}$

$V\left(G_{1} \times \mathrm{G}_{2}\right)=V_{1} \times V_{2}=V$ be the set of vertices of $G_{1}, G_{2}$ and $G_{1} \times G_{2}$ respectively. Since $n$ is power of a prime, we have $\gamma\left(G_{1}\right)=2$ (Theorem 1.2).

Let $D_{1}=\left\{u_{d_{1}}, u_{d_{2}}\right\}$ be a dominating set of $G_{1}$ with minimum cardinality 2 . Now in $G_{1} \times G_{2}$, consider the set of vertices $D=\left\{\left(u_{d_{1}}, p\right),\left(u_{d_{1}}, p^{j}\right),\left(u_{d_{2}}, p\right),\left(u_{d_{2}}, p^{j}\right)\right\}, j \neq 1$.

Let $(u, v)$ be any vertex of $V-D$ in $G_{1} \times G_{2}$. Then the vertex $u$ in $G_{1}$ is adjacent to either $u_{d_{1}}$ or $u_{d_{2}}$ in $D_{1}$. The vertex $v$ in $G_{2}$ is adjacent to either $p$ or $p^{j}$ according to $\operatorname{GCD}(v, p)=p$ or $\operatorname{GCD}\left(v, p^{j}\right)=p$. Thus by the definition of direct product, the vertex $(u, v)$ in $V-D$ is adjacent to either $\left(u_{d_{1}}, p\right)$ or $\left(u_{d_{1}}, p^{j}\right) ; \quad$ or $\left(u_{d_{2}}, p\right)$ or $\left(u_{d_{2}}, p^{j}\right)$ in $D$. Thus $D$ becomes a dominating set of $G_{1} \times G_{2}$.

We now show that $D$ is minimal. That is deletion of any vertex in $D$ does not make $D$, a dominating set any more. Suppose the vertex $\left(u_{d_{1}}, p\right)$ is deleted from $D$. We know that the degree of a vertex in $G_{1}$ is $\varphi(n)$. So let $u_{d_{1}}$ be adjacent to the vertices $u_{1}, u_{2}, \ldots . . u_{\varphi(n)}$ in $G_{1}$. 
Consider the set $S=\left\{\left(u_{1}, p^{k}\right),\left(u_{2}, p^{k}\right), \ldots,\left(u_{\varphi(n)}, p^{k}\right)\right\}$ in $G_{1} \times G_{2}$. Since $\operatorname{deg}_{G_{1}}\left(u_{d_{2}}\right)$ is $\varphi(n)$, all the vertices $u_{1}, u_{2}, \ldots ., u_{\varphi(n)}$ are not dominated by $u_{d_{2}}$. Otherwise the vertices of $V_{1}-\left\{u_{1}, u_{2}, \ldots . . u_{\varphi(n)}\right\}$ in $G_{1}$ are dominated neither by $u_{d_{1}}$ nor by $u_{d_{2}}$. So all the vertices in $S$ are not dominated by $\left(u_{d_{2}}, p\right)$ and $\left(u_{d_{2}}, p^{j}\right)$. Since GCD $\left(p^{j}, p^{k}\right) \neq$ $p$, all the vertices in $S$ are not dominated by $\left(u_{d_{1}}, p^{j}\right)$. Thus no vertex in $D-\left\{\left(u_{d_{1}}, p\right)\right\}$ can dominate the vertices of $S$. Thus $D-\left\{\left(u_{d_{1}}, p\right)\right\}$ is not a dominating set of $G_{1} \times G_{2}$. Similar is the case with the deletion of any other vertex in $D$. Thus $D$ becomes a minimal dominating set of $G_{1} \times G_{2}$.

Further if we form a dominating set of $G_{1} \times G_{2}$ in any other manner, then the order of such a set is not smaller than that of $D$. This is because of the properties of prime numbers. Thus the domination number of $G_{1} \times G_{2}$ is 4 .

That is $\quad \gamma\left(G_{1} \times G_{2}\right)=|D|=4$.

Theorem 2.3: The domination number of $G_{1} \times G_{2}$ is 6 , if $n=2 p$ where $p$ is an odd prime.

Proof: Let $n=2 p, p$ is an odd prime. Consider the graph $G_{1} \times G_{2}$.

$$
\text { Let } \begin{aligned}
V\left(G_{1}\right) & =\{0,1,2, \ldots \ldots ., 2 p-1\}=V_{1} \\
V\left(G_{2}\right) & =\{2, p, 2 p\}=V_{2} \text { and } \\
V\left(G_{1} \times \mathrm{G}_{2}\right) & =V_{1} \times V_{2}=V
\end{aligned}
$$

be the set of vertices of the graphs $G_{1}, G_{2}$ and $G_{1} \times G_{2}$ respectively.

Consider a dominating set $D_{1}$ of $G_{1}$ with cardinality 2, given by $D_{1}=\left\{u_{d_{1}}, u_{d_{2}}\right\}$ where $\left|u_{d_{1}}-u_{d_{2}}\right|=p$,

(Theorem 1.2 ). To obtain a dominating set of $G_{1} \times G_{2}$, consider the set $D$ of vertices in $G_{1} \times G_{2}$ given by $D=$ $\left\{\left(u_{d_{1}}, 2\right),\left(u_{d_{1}}, p\right),\left(u_{d_{1}}, 2 p\right)\right.$,

$\left.\left(u_{d_{2}}, 2\right),\left(u_{d_{2}}, p\right),\left(u_{d_{2}}, 2 p\right)\right\}$ We now prove that $D$ is a dominating set of $G_{1} \times G_{2}$ as follows.

Let $(u, v)$ be any vertex in $V-D$ in $G_{1} \times G_{2}$. Then the vertex $u$ in $G_{1}$ is adjacent to either $u_{d_{1}}$ or $u_{d_{2}}$ in $D_{1}$. Since $n=2 p$, there are no isolated vertices in $G_{2}$. Hence the vertex $v$ in $G_{2}$ is adjacent to at least one vertex of $\{2, p, 2 p\}$ in $G_{2}$. Thus by the definition of direct product, the vertex $(u, v)$ in $V-D$ is adjacent to either $\left(u_{d_{1}}, 2\right)$ or $\left(u_{d_{1}}, p\right)$ or $\left(u_{d_{1}}, 2 p\right) ;\left(u_{d_{2}}, 2\right)$ or $\left(u_{d_{2}}, p\right)$ or $\left(u_{d_{2}}, 2 p\right)$ in $D$. Since $(u, v)$ is an arbitrary vertex in $V-D$ it follows that $D$ is a dominating set of $G_{1} \times G_{2}$.

We now show that $D$ is minimal. For this consider any vertex in $D$, say $\left(u_{d_{1}}, p\right)$. Suppose we delete this vertex from $D$. Here the vertex $u_{d_{1}}$ is non-adjacent to $u_{d_{2}}$, because $\mid u_{d_{1}}$ $u_{d_{2}} \mid=p$ and hence GCD $\left(u_{d_{1}}-u_{d_{2}}, n\right) \neq 1$. This implies that the vertex $\left(u_{d_{1}}, p\right)$ is non-adjacent to the vertices $\left(u_{d_{2}}, 2\right),\left(u_{d_{2}}, p\right),\left(u_{d_{2}}, 2 p\right)$. Also $\left(u_{d_{1}}, p\right)$ is non-adjacent to the vertices $\left(u_{d_{1}}, 2\right),\left(u_{d_{1}}, 2 p\right)$ by the definition of direct product. Thus the vertex $\left(u_{d_{1}}, p\right)$ in $V-D$ is not adjacent to any vertex of $D-\left\{\left(u_{d_{1}}, p\right)\right\}$. That is, $D-$ $\left\{\left(u_{d_{1}}, p\right)\right\}$ is not a dominating set. Similar is the case with the deletion of any other vertex in $D$. Hence $D$ is a minimal dominating set of $G_{1} \times G_{2}$. Further if we form a dominating set of $G_{1} \times G_{2}$ in any other manner, then the order of such a set is bigger than that of $D$ due to the properties of prime numbers.

Therefore $\gamma\left(G_{1} \times G_{2}\right)=|D|=6$.

Theorem 2.4: If $n \neq p^{\alpha}, \quad n \neq 2 p$ and $n=$ $p_{1}^{\alpha_{1}} p_{2}^{\alpha_{2}} \ldots \ldots . p_{k}^{\alpha_{k}}$ where $\alpha_{i} \geq 1$, then the domination number of $G_{1} \times G_{2}$ is given by $\gamma\left(G_{1} \times G_{2}\right)=(\lambda+1) k$, where $\lambda$ is the length of the longest stretch of consecutive integers in $V_{1}$ of $G_{1}$ each of which shares a prime factor with $n$ and $k$ is the core of $n$.

Proof: Let $n \neq p^{\alpha}, n \neq 2 p$ and $n=p_{1}^{\alpha_{1}} p_{2}^{\alpha_{2}} \ldots \ldots . p_{k}^{\alpha_{k}}$ where $\alpha_{i} \geq 1$. Then by Theorem 1.4, $\gamma\left(G_{1}\right)=\lambda+1$.

Let $D_{1}=\left\{u_{d_{1}}, u_{d_{2}}, \ldots \ldots ., u_{d_{\lambda+1}}\right\}$ be a dominating set of $G_{1}$ with minimum cardinality $\lambda+1$. Again by Theorem 1.10 , we know that $\gamma_{t}\left(G_{2}\right)=k$ where $k$ is the core of $n$. Let $D_{2}=\left\{p_{1}, p_{2}, \ldots \ldots p_{k-1}, p_{1} p_{2} \ldots p_{k}\right\} \quad$ be a total dominating set of $G_{2}$ with minimum cardinality $k$.

Now consider the Cartesian product of the sets $D_{1}$ and $D_{2}$ as follows.

$$
\begin{aligned}
& D=D_{1} \times D_{2} \\
& =\left\{u_{d_{1}}, u_{d_{2}}, \ldots \ldots ., u_{d_{\lambda+1}}\right\} \times\left\{p_{1}, p_{2}, \ldots \ldots p_{k-1}, p_{1} p_{2} \ldots p_{k}\right\} \\
& =\left\{\left(u_{d_{1}}, p_{1}\right), \ldots \ldots, \quad\left(u_{d_{1}}, p_{k-1}\right), \quad\left(u_{d_{1}}, p_{1} p_{2} \ldots p_{k}\right),\right. \\
& \left(u_{d_{2}}, p_{1}\right), \ldots, \ldots\left(u_{d_{2}}, p_{k-1}\right), \quad\left(u_{d_{2}}, p_{1} p_{2} \ldots p_{k}\right), \\
& \vdots \quad \vdots \quad \vdots \\
& \left.\left(u_{d_{\lambda+1}}, p_{1}\right), \ldots \ldots\left(u_{d_{\lambda+1}}, p_{k-1}\right),\left(u_{d_{\lambda+1}}, p_{1} p_{2} \ldots p_{k}\right)\right\} .
\end{aligned}
$$

Let $(u, v)$ be any vertex of $V-D$ in $G_{1} \times G_{2}$. Then vertex $u$ is adjacent to some vertex $u_{d_{l}}$ in $D_{1}$ and vertex $v$ is adjacent to either $p_{j}$, for $1 \leq j \leq k-1$ or $p_{1} p_{2} \ldots p_{k}$ as $D_{1}$ and $D_{2}$ are dominating sets of $G_{1}$ and $G_{2}$ respectively. That is vertex $(u, v)$ is adjacent to $\left(u_{d_{l}}, p_{j}\right)$ or $\left(u_{d_{l}}, p_{1} p_{2} \ldots p_{k}\right)$ in $D$.

Thus $D=D_{1} \times D_{2}$ becomes a dominating set of $G_{1} \times$ $G_{2}$. We now prove that deletion of any vertex in $D$ does not make the resulting set a dominating set any more.

Let $\left(u_{x}, v_{y}\right) \in D$. Then $u_{x}$ is any one of the vertices $u_{d_{1}}, u_{d_{2}}, \ldots \ldots \ldots, u_{d_{\lambda+1}}$ and $v_{y}$ is any one of the vertices $p_{1}, p_{2}, \ldots \ldots p_{k-1}, p_{1} p_{2} \ldots p_{k}$. Let $u_{x}=u_{d_{i}}, 1 \leq i \leq \lambda+1$. Suppose $u_{x}$ is adjacent to the vertices $u_{1}, u_{2}, \ldots \ldots, u_{\varphi(n)}$ in $G_{1}$. To select the vertex $v_{y}$, we proceed as follows.

Case 1: Suppose $v_{y}=p_{m}$ where $1 \leq m \leq k-1$. Then $v_{y}$ is adjacent to $p_{m} p_{k}$, as $\operatorname{GCD}\left(p_{m} p_{k}, p_{m}\right)=p_{m}$. So by the definition of direct product $\left(u_{x}, v_{y}\right)=\left(u_{d_{i}}, p_{m}\right)$ is adjacent to the vertices $\left(u_{1}, p_{m} p_{k}\right),\left(u_{2}, p_{m} p_{k}\right), \ldots .,\left(u_{\varphi(n)}\right.$, $\left.p_{m} p_{k}\right)$.

Suppose we delete vertex $\left(u_{x}, v_{y}\right)=\left(u_{d_{i}}, p_{m}\right)$ from $D$. If we consider the set of vertices $\left\{\left(u_{1}, p_{m} p_{k}\right),\left(u_{2}\right.\right.$, $\left.\left.p_{m} p_{k}\right), \ldots,\left(u_{\varphi(n)}, p_{m} p_{k}\right)\right\}$ in $V-D$ of $G_{1} \times G_{2}$, then no vertex in this set is adjacent to a vertex in $D-\left(u_{x}, v_{y}\right)$. This is because vertex $p_{m} p_{k}$ is not adjacent to any vertex $p_{j}$ or vertex $p_{1} p_{2} \ldots p_{k}$ as

$\operatorname{GCD}\left(p_{m} p_{k}, p_{j}\right)=1$ for $m \neq j, \quad 1 \leq m, j \leq k-1$ and GCD $\left(p_{m} p_{k}, p_{1} p_{2} \ldots p_{k}\right)=p_{m} p_{k}$. This means that $D-$ $\left\{\left(u_{x}, v_{y}\right)\right\}$ is not a dominating set. 
Case 2: Suppose $v_{y}=p_{1} p_{2} \ldots p_{k}$. Then $v_{y}$ is adjacent to $p_{k}$, as $\operatorname{GCD}\left(p_{1} p_{2} \ldots p_{k}, p_{k}\right)=p_{k}$. Hence in this case, by the definition of direct product $\left(u_{x}, v_{y}\right)=\left(u_{d_{i}}, p_{1} p_{2} \ldots p_{k}\right)$ is adjacent to the vertices $\left(u_{1}, p_{k}\right),\left(u_{2}, p_{k}\right), \ldots,\left(u_{\varphi(n)}\right.$, $\left.p_{k}\right)$.

Suppose we delete vertex $\left(u_{x}, v_{y}\right)=\left(u_{d_{i}}, p_{1} p_{2} \ldots p_{k}\right)$ from $D$. Then the set $\left\{\left(u_{1}, p_{k}\right),\left(u_{2}, p_{k}\right), \ldots .,\left(u_{\varphi(n)}, p_{k}\right)\right\}$ in $V-D$ of $G_{1} \times G_{2}$ is such that no vertex in this set is adjacent to a vertex of $D-\left(u_{x}, v_{y}\right)$ because

$\operatorname{GCD}\left(p_{k}, p_{j}\right)=1$ for $1 \leq j \leq k-1$. This implies that $D-\left(u_{x}, v_{y}\right)$ is not a dominating set. . Thus $D$ becomes a minimal dominating set. Further if we form a dominating set of $G_{1} \times G_{2}$ in any other manner, then the order of such a set is not smaller than that of $D$. This is due to the properties of prime numbers.

Therefore $\gamma\left(G_{1} \times G_{2}\right)=|D|=(\lambda+1) \cdot k$.

\section{TOTAL DOMINATION IN DIRECT PRODUCT GRAPH $G_{1} \times G_{2}$}

In this section the results on the total dominating sets of direct product graph $G_{1} \times G_{2}$ are discussed for different values of $n$.

$G_{1} \times G_{2}$ is a completely disconnected graph on $n$ vertices, if $n$ is a prime. So there are no edges between these $n$ vertices. Therefore total dominating set does not exist for $G_{1} \times G_{2}$ when $n$ is a prime.

Theorem 3.1: The total domination number of $G_{1} \times G_{2}$ is 4 , if $n$ is power of a prime.

Proof: Let $n=p^{\alpha}, \alpha>1$. Consider the graph $G_{1} \times G_{2}$. By Theorem 2.2 we know that $\gamma\left(G_{1} \times G_{2}\right)=4$ for $n=p^{\alpha}$. So we have $\gamma_{t}\left(G_{1} \times G_{2}\right) \geq 4$. To get a total dominating set of $G_{1} \times G_{2}$, we proceed along the lines of Theorem 2.2 and hence $T=\left\{\left(u_{d_{1}}, p\right),\left(u_{d_{1}}, p^{j}\right),\left(u_{d_{2}}, p\right),\left(u_{d_{2}}, p^{j}\right)\right\}$, $\mathrm{j}>1$ is a dominating set of $G_{1} \times G_{2}$ with minimum cardinality.

We now show that $T$ is a total dominating set by showing that the vertices in $T$ dominate among themselves. Since $u_{d_{1}}$, $u_{d_{2}}$ are consecutive integers, we have GCD $\left(u_{d_{1}}-u_{d_{2}}\right.$, $n)=1$ and hence by the definition of adjacency in $G_{1}$, vertices $u_{d_{1}}, u_{d_{2}}$ are adjacent to each other.

Further for $\mathrm{j}>1$, we have $\operatorname{GCD}\left(p, p^{j}\right)=p$. Hence by the definition of adjacency in $G_{2}$, vertices $p$ and $p^{j}$ are adjacent to each other. Therefore by the definition of direct product, $\left(u_{d_{1}}, p\right),\left(u_{d_{2}}, p^{j}\right) ;\left(u_{d_{2}}, p\right),\left(u_{d_{1}}, p^{j}\right)$ are the pairs of adjacent vertices in $T$. Thus $T$ becomes a total dominating set with minimum cardinality.

Therefore $\gamma_{t}\left(G_{1} \times G_{2}\right)=4$.

Theorem 3.2: If $n$ is not power of a single prime and $n=p_{1}^{\alpha_{1}} p_{2}^{\alpha_{2}} \ldots \ldots . . p_{k}^{\alpha_{k}}$, where $\alpha_{i} \geq 1, k>1$, then the total domination number of $G_{1} \times G_{2}$ is given by $\gamma_{t}\left(G_{1} \times\right.$ $\left.G_{2}\right)=(\lambda+1) \cdot k$, where $\lambda$ is the length of the longest stretch of consecutive integers in $V_{1}$ of $G_{1}$ each of which shares a prime factor with $n$ and $k$ is the core of $n$.

Proof: Let $n=p_{1}^{\alpha_{1}} p_{2}^{\alpha_{2}} \ldots \ldots . . p_{k}^{\alpha_{k}}$, where $\alpha_{i} \geq 1, k>1$.
By Theorem 2.4, we know that $\gamma\left(G_{1} \times G_{2}\right)=(\lambda+1) \cdot k$ where $\lambda$ is the length of the longest stretch of consecutive integers in $V_{1}$ of $G_{1}$ each of which shares a prime factor with $n$ and $k$ is the core of $n$.

Therefore $\gamma_{t}\left(G_{1} \times G_{2}\right) \geq(\lambda+1) \cdot k$

Without loss of generality, we take

$$
\begin{aligned}
& T=\left\{u_{d_{1}}, u_{d_{2}}, \ldots \ldots ., u_{d_{\lambda+1}}\right\} \\
& \times\left\{p_{1}, p_{2}, \ldots \ldots p_{k-1}, p_{1} p_{2} \ldots p_{k}\right\} \\
& =\left\{\left(u_{d_{1}}, p_{1}\right), \quad \ldots \ldots ., \quad\left(u_{d_{1}}, p_{k-1}\right), \quad\left(u_{d_{1}}, p_{1} p_{2} \ldots p_{k}\right),\right. \\
& \left(u_{d_{2}}, p_{1}\right), \ldots \ldots \ldots .,\left(u_{d_{2}}, p_{k-1}\right), \quad\left(u_{d_{2}}, p_{1} p_{2} \ldots p_{k}\right), \\
& \vdots \quad \vdots \quad \vdots \\
& \left.\left(u_{d_{\lambda+1}}, p_{1}\right) \ldots \ldots .,\left(u_{d_{\lambda+1}}, p_{k-1}\right),\left(u_{d_{\lambda+1}}, p_{1} p_{2} \ldots p_{k}\right)\right\}
\end{aligned}
$$

where $u_{d_{1}}, u_{d_{2}}, \ldots \ldots ., u_{d_{\lambda+1}}$ are consecutive integers and $p_{1}, p_{2}, \ldots \ldots p_{k-1}, p_{1} p_{2} \ldots p_{k}$ are prime factors of $n$. Then as in Theorem 2.4, we can show that $T$ is a dominating set of $G_{1} \times G_{2}$ with minimum cardinality.

We now show that vertices in $T$ are dominated by the vertices of $T$. Since $u_{d_{1}}, u_{d_{2}}, \ldots \ldots, u_{d_{\lambda+1}}$ are consecutive integers, each vertex $u_{d_{i}}$ for $1 \leq i \leq \lambda$ is adjacent to its succeeding vertex $u_{d_{i+1}}$, as $\operatorname{GCD}\left(u_{d_{i+1}}-u_{d_{i}}, n\right)=1$. Further $\operatorname{GCD}\left(p_{j}, p_{1} p_{2} \ldots p_{k}\right)=p_{j}$, for $1 \leq j \leq k-1$, implies that each one of $p_{1}, p_{2}, \ldots \ldots p_{k-1}$ is adjacent to $p_{1} p_{2} \ldots p_{k}$. Hence by the nature of adjacency in direct product, each vertex $\left(u_{d_{i}}, p_{j}\right)$ in $T$ is adjacent to $\left(u_{d_{i+1}}, p_{1} p_{2} \ldots p_{k}\right)$ in $T$. This implies that the vertices in $T$ are dominated by the vertices of $T$. Thus $T$ becomes a total dominating set of $G_{1} \times G_{2}$.

Since $\gamma\left(G_{1} \times G_{2}\right)=(\lambda+1) \cdot k$, and $|T|=(\lambda+1) \cdot k$, it follows that $T$ is a total dominating set of $G_{1} \times G_{2}$ with minimum cardinality.

Therefore $\gamma_{t}\left(G_{1} \times G_{2}\right)=(\lambda+1) \cdot k$.

\section{ILLUSTRATIONS}

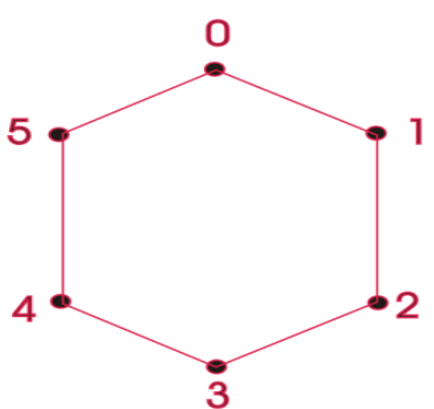

Fig. 1 


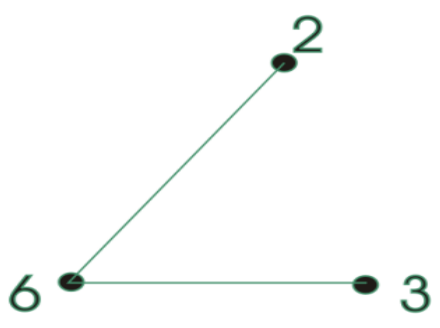

Fig. 2

$\mathbf{G}_{2}=\mathbf{G}\left(\mathbf{V}_{6}\right)$

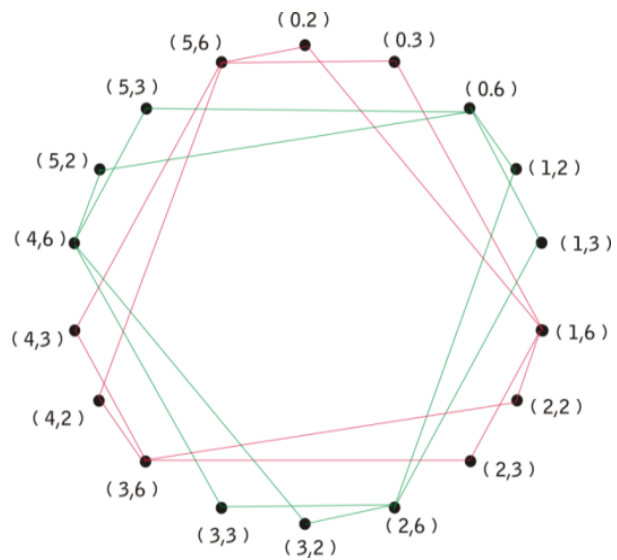

Fig. 3

$\boldsymbol{G}_{\mathbf{1}} \times \boldsymbol{G}_{\mathbf{2}}$ is a disconnected graph

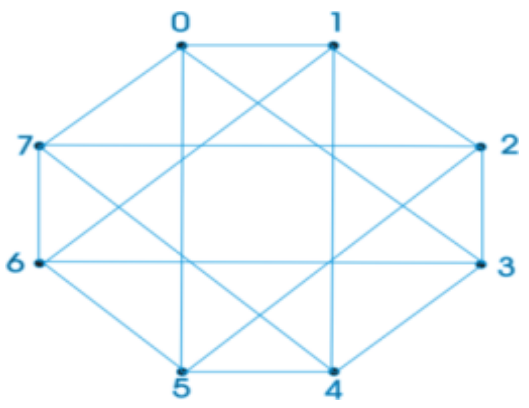

Fig. 4

$$
G_{1}=G\left(Z_{8}, \varphi\right)
$$

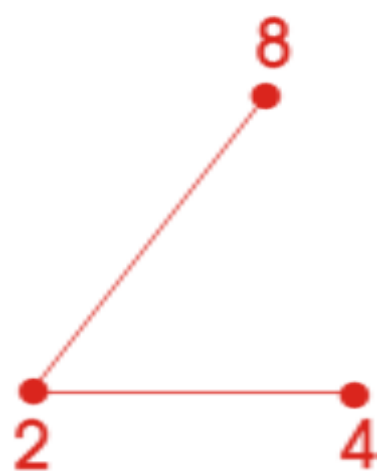

Fig. 5

$G_{2}=G\left(V_{8}\right)$

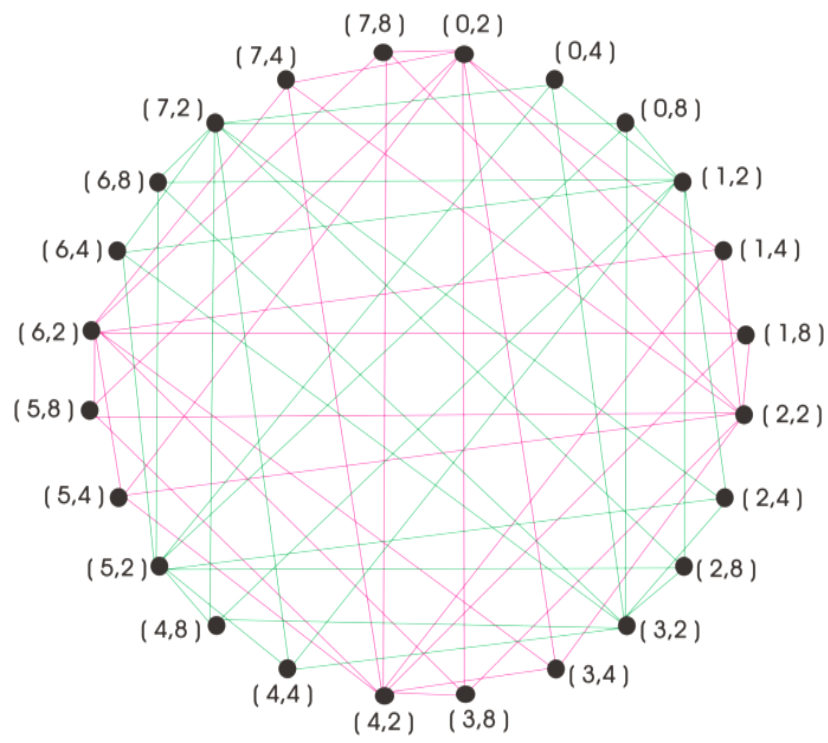

Fig. 6

$\boldsymbol{G}_{\mathbf{1}} \times \boldsymbol{G}_{\mathbf{2}}$ is a disconnected graph

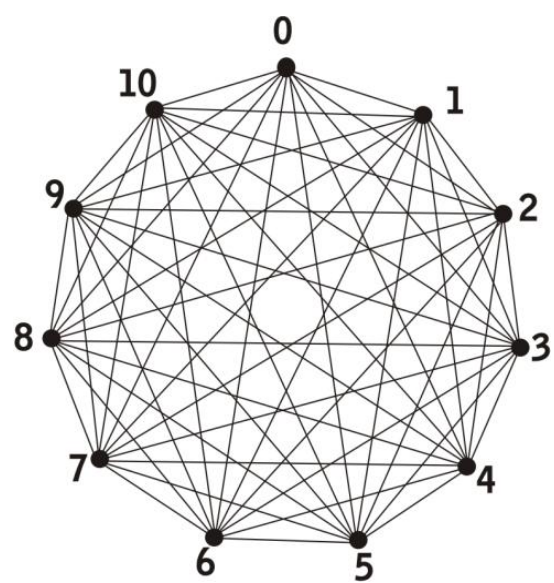

Fig. 7

$G_{1}=G\left(Z_{11}, \varphi\right)$ 
Fig. 8

$G_{2}=G\left(V_{11}\right)$

Fig. 9

$\boldsymbol{G}_{\mathbf{1}} \times \boldsymbol{G}_{\mathbf{2}}$ is a completely disconnected graph

Table 1. Dominations in Direct Product Graph $G_{1} \times G_{2}$

\begin{tabular}{|c|c|c|c|c|c|}
\hline $\begin{array}{c}\mathbf{n} \\
\text { Values }\end{array}$ & Dominating sets & $G_{1}=\mathbf{G}\left(\mathbf{Z}_{\mathbf{n}}, \varphi\right)$ & $G_{2}=G\left(V_{n}\right)$ & $G_{1} \times G_{2}$ & $\begin{array}{c}\text { Domination } \\
\text { Number in } \\
G_{1} \times G_{2}\end{array}$ \\
\hline \multirow{2}{*}{$n=6$} & $\begin{array}{c}\text { Minimum } \\
\text { Dominating set }\end{array}$ & $\{0,3\}$ & $\{6\}$ & $\begin{array}{c}\{(0,2),(0,3),(0,6),(3,2) \\
(3,3),(3,6)\}\end{array}$ & $\gamma=6$ \\
\hline & $\begin{array}{l}\text { Minimum Total } \\
\text { Dominating set }\end{array}$ & $\{0,1,3,4\}$ & $\{2,6\}$ & $\begin{array}{l}\{(0,2),(0,6),(1,2),(1,6), \\
(3,2),(3,6),(4,2),(4,6)\}\end{array}$ & $\gamma_{t}=8$ \\
\hline \multirow{2}{*}{$n=8$} & $\begin{array}{c}\text { Minimum } \\
\text { Dominating set }\end{array}$ & $\{0,1\}$ & $\{2\}$ & $\{(0,2),(0,8),(1,2),(1,8)\}$ & $\gamma=4$ \\
\hline & $\begin{array}{l}\text { Minimum Total } \\
\text { Dominating set }\end{array}$ & $\{0,1\}$ & $\{2,8\}$ & $\{(0,2),(0,8),(1,2),(1,8)\}$ & $\gamma_{t}=4$ \\
\hline \multirow{2}{*}{$n=11$} & $\begin{array}{c}\text { Minimum } \\
\text { Dominating set }\end{array}$ & $\{0\}$ & $\{11\}$ & Vertex set of $G_{1} \times G_{2}$ & $\gamma=11$ \\
\hline & $\begin{array}{l}\text { Minimum Total } \\
\text { Dominating set }\end{array}$ & $\{0,1\}$ & Does not exist & Null graph & $\gamma_{t}$ does not exist \\
\hline
\end{tabular}

\section{REFERENCES}

[1] Nathanson and Melvyn B . Connected components of arithmetic graphs, Monat. fur. Math, 29, $219 \quad-$ 220(1980).

[2] Cockayne, C.J., Dawes, R.M. and Hedetniemi,S.T.Total domination in graphs, Networks, 10, 211-219 (1980).

[3] Cockayne, E.J. and Hedetniemi, S.T. Towards a theory of domination in graphs, Networks, 7, 247 - 261 (1977).
[4] Whitehead, A.N. and Russel, B. Principia Mathematica, Volume 2, Cambridge University, Press, Cambridge (1912).

[5] Weichsel, P.M. The Kronecker product of graphs, Proc. Amer. Math.Soc., 13, 47-52, (1962).

[6] Imrich, W. Factoring cardinal product graphs in polynomial time, Discrete Math., 192, 119-144(1998).

[7] Hammack, R, Imrich, W and Klavzar, S. Handbook of product graphs, CRC Press, (2011). 
[8] S.Uma Maheswari, Some studies on the product graphs of Euler Totient Cayley graphs and Arithmetic $V_{n}$ graphs, Ph.D. Thesis submitted to S.P. Women's University, Tirupati, India, (2012).

[9] Madhavi, L. Studies on domination parameters and enumeration of cycle in some Arithmetic graphs, Ph. D. Thesis submitted to S.V.University, Tirupati, India, (2002).

[10] S.Uma Maheswari and B.Maheswari, Domination parameters of Euler Totient Cayley graphs, Rev.Bull.Cal.Math.Soc., 19,(2),207-214(2011).
[11] Vasumathi, N. Number theoretic graphs, Ph. D. Thesis submitted to S.V.University, Tirupati, India, (1994).

[12] S.Uma Maheswari and B.Maheswari, Some domination parameters of Arithmetic graph $V_{n}$, IOSRJM, 2,(6), 1418 (2012)

[13] S.Uma Maheswari and B.Maheswari, Some properties of direct product graphs of Cayley graphs with Arithmetic graphs, 54, (14), 34-37,(2012). 